\title{
Modelling of a New Compton Imaging Modality for an In-Depth Characterisation of Flat Heritage Objects
}

\author{
Patricio Guerrero $^{1,2,3}$ Mai K. Nguyen ${ }^{2}$ Laurent Dumas ${ }^{3} \quad$ Serge X. Cohen $^{1}$ \\ ${ }^{1}$ IPANEMA USR 3461, CNRS/MCC/UVSQ/MNHN, Gif-sur-Yvette, France, \\ \{patricio.guerrero-prado, serge.cohen\} @university.org \\ ${ }^{2}$ Equipes de Traitement de l'Information et Systèmes UMR 8051, ENSEA/UCP/CNRS \\ mai.nguyen-vergerau-cergy . fr \\ ${ }^{3}$ Laboratoire de Mathématiques de Versailles UMR 8100, UVSQ/CNRS, Versailles, France, \\ laurent.dumas@uvsq.fr
}

\begin{abstract}
Objects having a flattened geometry, such as those encountered in heritage, have always been difficult to be analysed with conventional X-ray tomography methods due to their anisotropic morphology. To overcome the limitations of classical tomography for such samples, we envisage a new imaging modality based on Compton scattering. While Compton effect is usually considered as noise in tomography, in Compton scattering tomography the conditions are set such that this becomes the imaging agent of the image formation process. Our interests are, firstly, to avoid the relative rotation between the object, the source and the detector, and secondly, to be able to obtain in-depth data even when the sample is supported by some deep or dense material by exploiting only back-scattered photons. To replace the information provided by multiple projections angles in classical tomography, we make use of the relation between the energy loss of the scattered photons and its scattering angle, the Compton equation. Modelling of this new modality, image formation and object reconstruction through a filtered back-projection algorithm of a Radon transform on a half-space is presented. The feasibility of this concept is supported by numerical simulations.
\end{abstract}

Keywords: Radon transform, Compton scattering tomography, image reconstruction

\section{Introduction}

\subsection{Context}

Analysis of flat objects has remained particularly challenging with X-ray imaging methods. X-ray imaging has the strong advantage of providing non-invasive/nondestructive probing of the material and enables 2D twodimensional (2D) imaging of the sample which is often mandatory when analysing heritage samples. However, a large set of those samples has a complex flattened geometry, i.e., a large ratio between the front area and its thickness and the high differential light path in distinct directions prohibits the relative rotations of the sample to perform a three-dimensional reconstruction as would be done in regular tomography, using either absorption or phase contrast modality. Such samples could be encountered in conservation/restoration studies of easel painting requiring the characterisation of the stratigraphical assemblage of pigments often over a very dense background layer (Figure 1), e.g. made of white lead. Another type of samples falling in this set is encountered in palaeontology with the Lagerstätten fossils (Gueriau et al., 2014) which are mechanically flattened during the fossilisation process and stand on one side of a thick sedimental slab which can not be thinned for the study (Figure 2). In both cases the volume of interest forms a layer on top of a material matrix which is opaque to X-ray either due to its density or its thickness.

In both cases scientists left with one of two choices : either to perform a stratigraphical section of the sample which is an invasive method, or perform a $2 \mathrm{D}$ analysis, for example with synchrotron X-ray fluorescence spectral raster-scanning (Gueriau et al., 2014) while an in-depth reconstruction is still desired. Furthermore, because of the opaque supporting material, transmission and forward scattering data, with a scattering angle inferior of $\frac{\pi}{2}$, are impossible to collect. This leads us to propose a modality tapping on back-scattered data, that is, data collected with a scattering angle comprised between $\frac{\pi}{2}$ and $\pi$, as shown in Figure 3.

Using the approximation that all electrons of the material are both free and at rest, the Compton equation establishes in (1) a diffeomorphism between the scattering angle and the scattered energy, provided that the incident beam is monochromatic, i.e. a single value $E_{0}$ of an incident energy is illuminating the object. In such circumstances the Compton scattering image formation process corresponds to a Radon transform over either one branch of a V-line or equivalently over lines in a half-space (Morvidone et al., 2010; Truong and Nguyen, 2011, 2015) when the scene is purely 2-dimensional, or over the surface of a cone (Nguyen et al., 2005; Cebeiro et al., 2015) when a 3-dimensional scene is considered. 


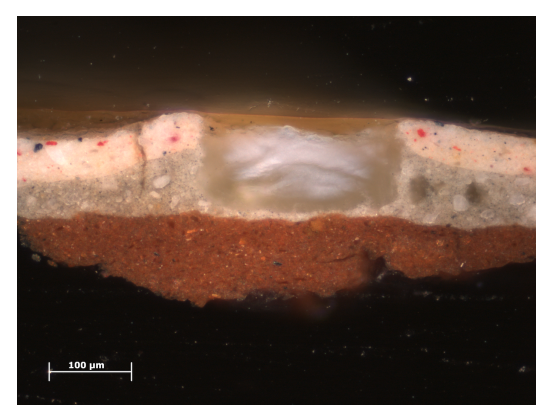

Figure 1. Paint cross-section showing a stratigraphical assemblage of The Anatomy Lesson of Dr. Nicolaes Tulp, 1632 by Rembrandt, Mauritshuis, The Hague. (c) Sample taken and prepared as cross-section by P. Noble during the conservation treatment of the painting in 1997, and re-photographed by A. van Loon, Mauritshuis, in 2010 for the Rembrandt Database.

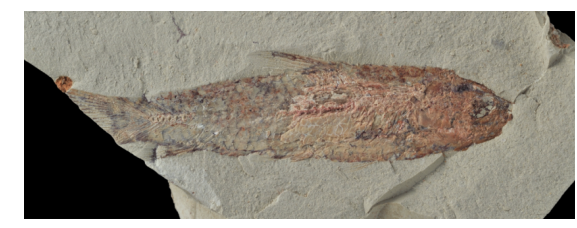

Figure 2. A flat fossil actinopterygian over a high thick X-ray absorbing support from the Kem Kem Beds in Morocco dated back to the Lower Cretaceous (95 million years ago). (C) P. Gueriau $(\mathrm{MHNM} / \mathrm{MNHN})$.

\subsection{Compton scattering tomography (CST)}

Three basic photon-matter interaction phenomena are considered in classical X-ray imaging and hence tomography : Photoelectric absorption, Rayleigh scattering which is both elastic and coherent, and Compton scattering which conversely is both inelastic and incoherent. In this last one, an incident photon of energy $E_{0}$ is absorbed by a target electron, who re-emits a secondary photon scattered by an angle $\omega$ relative to the direction of the original photon. The scattered photon has then an energy $E_{\omega}$ which is related to the scattering angle $\omega$ by (1), the Compton equation, when the target electron is both free and at rest.

In classical X-ray imaging and tomography, Compton scattered signal is considered as noise added to photoelectric absorption and coherent scattered data. This is because X-ray transmission signal is dominated by the photoelectric absorption whilst coherent scattering may produce significant amplitude variations at low scattering angles thanks to constructive and destructive interference effects due to the coherent nature of this scattering. However, depending on the material, if the incident radiation has an energy superior of $40-50 \mathrm{keV}$, Compton scattering is becoming the dominant phenomenon in the process, even more when detection is performed far from the transmission geometry $(\omega=0)$.

Classical tomographic imaging modalities, developed and used in most all applications in the last half century include : Transmission Computed Tomography (CT), Single Photon Emission Tomography (SPECT) and Positron
Emission Tomography (PET). All of them regard primary radiation and perform a $3 \mathrm{D}$ mapping leaning on relative rotations between the object and the imaging setup. In such framework the Compton scattering is adding a nonuniform background to the observation, a systematic bias which leads to artefacts in the reconstruction if it is not accounted for. As the relative importance of the Compton scattering over the other two processes mentioned above is increasing with an increase on incident energy, its effect is even more important when using higher energy $\gamma$-ray imaging and tomography.

The idea of exploiting scattered radiation by Compton effect in imaging techniques has been introduced and studied simultaneously and it has given birth to CST (Norton, 1994; Nguyen et al., 2005; Morvidone et al., 2010; Cebeiro et al., 2015; Truong and Nguyen, 2011, 2015), which focuses to reconstruct the electron density map of the object.

To replace the information provided by multiple projections angles in classical transmission or emission tomography, CST exploits the energy loss of the scattered photons. This energy loss encodes the scattering angle information thanks to the Compton equation given by

$$
E_{\omega}=\frac{E_{0}}{1+\frac{E_{0}}{m_{e} c^{2}}(1-\cos \omega)},
$$

where $m_{e} c^{2}=511 \mathrm{keV}$ is the rest mass energy of the electron.

The first CST scanner was proposed in 1994 (Norton, 1994) through a Radon transform over arc of circles starting at a $\gamma$-ray source and ending at the detecting point. Radon transforms over V-lines and its analytic inversion formula as well as a filtered back-projection reconstruction was proposed in (Morvidone et al., 2010), three different types of these V-line transforms with fixed axis direction are studied in (Truong and Nguyen, 2011) and new properties and applications including a Radon transform on a half-space were presented recently in (Truong and Nguyen, 2015). Radon transforms over conical surfaces having fixed axis directions and variable opening angle are studied in (Nguyen et al., 2005) where an analytic inversion formula is proposed, with applications to emission imaging based on Compton scattered radiation. A backprojection inversion for this conical Radon transform was developed recently in (Cebeiro et al., 2015).

This paper is organised as follows. Section 2 describes this new CST modality, and presents image formation process as well as object reconstructions via a back-projection algorithm related to a half-space Radon transform in the framework of a two dimensional setup with back-scattering. In Section 3 we present numerical scheme and some simulation results for flat objects both for energy resolved image formation and sample reconstruction. Finally, Section 4 closes this paper with a conclusion and perspectives. 
The three dimensional case, corresponding to the conical Radon transform, will not be presented due to fact that the azimuthal scattering angle is uniformly distributed in a Compton event, that is to say, the angular distribution of scattered photons has axial symmetry around the direction of incidence.

\section{Modelling of the new CST modality}

CST focuses to reconstruct the electron density map of the object. In this paper, this density will be represented by a nonnegative bump function (both smooth and of bounded support) $f: \mathbb{R}^{+} \times \mathbb{R} \rightarrow \mathbb{R}^{+}$.

Fundamentally, an incident photon of energy $E_{0}$, coming from a point source $\mathbf{S}$, is inelastically scattered by an electron located inside the object at $\mathbf{M}$ subtending an angle $\omega\left(\frac{\pi}{2}<\omega<\pi\right)$ with the direction of incidence. The scattered photon of energy $E_{\omega}$, approximated by (1), arrives finally at a detecting point $\mathbf{D}$. In this paper, $\mathbf{D}$ is located over the $O y$-axis of the Cartesian reference plane and noted $\mathbf{D}_{\zeta}=(0, \zeta)$. Obviously we will have data for values of $\zeta$ verifying $|\zeta|>\zeta_{0}$ for some $\zeta_{0}>0$. The parallel beam is centred at the $O x$-axis. See Figs. 3 and 4 for more details.

As already mentioned, in this paper we will considerer a two dimensional setup, i.e., a slice of a three dimensional space through the $z$-axis.

\subsection{Proposed setup by back-scattering}

A synchrotron radiation setup with a monochromatic parallel X-ray beam (about $50 \mathrm{keV}$ ) and a space-energy resolved detector is proposed. As represented in Figure 3, the detector will be placed between the source and the object to capture back-scattered photons. It will have then a hole in the middle of length $2 \zeta_{0}$ to allow the beam to go through. Vertical translations of the sample will allow the imaging of the full sample despite the limited beam size.

This configuration is considered to overcome limitations of getting data through classical methods mentioned before.

In Figure 4, scattered radiation arriving to a detecting point $\mathbf{D}_{\zeta}$ laying on the line that crosses both $\mathbf{M}_{1}$ and $\mathbf{D}_{\zeta}$ will have an energy loss related to $\omega_{1}$, for example radiation scattered from $\mathbf{M}_{3}$. The same will occur related to $\mathbf{M}_{2}$ and $\omega_{2}$. One will be able to identify, for example, points $\mathbf{M}_{1}$ and $\mathbf{M}_{2}$ radiated with the same X-ray beam at the same time through energy loss data.

\subsection{Direct problem : Image formation}

\subsubsection{The half-space Radon transform (HRT)}

The half-space Radon transform and its analytic inverse formula were introduced in (Truong and Nguyen, 2015). Briefly, it is a Radon transform, introduced in (Radon, 1917), defined on half-lines, starting on a detecting point towards the direction of where the sample is placed. The interest is that it performs an integration of the object, in this case a function representing an electron density distri-

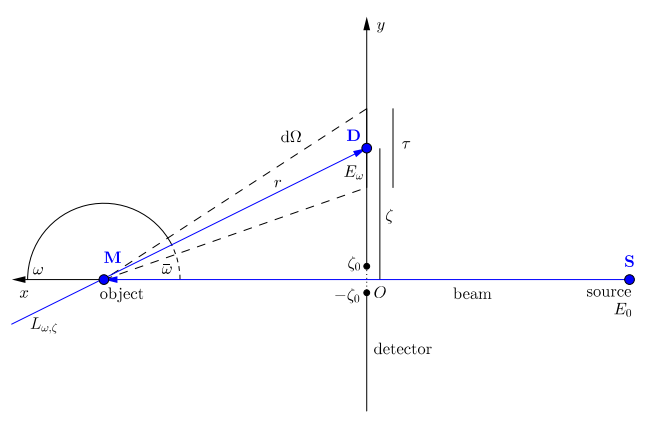

Figure 3. Simulated setup. Photons coming from a source at $\mathbf{S}$ are back-scattered at $\mathbf{M}$ by an angle $\omega$ into the solid angle $\mathrm{d} \Omega$. They are finally detected at $\mathbf{D}$, who lays on the $O y$-axis.

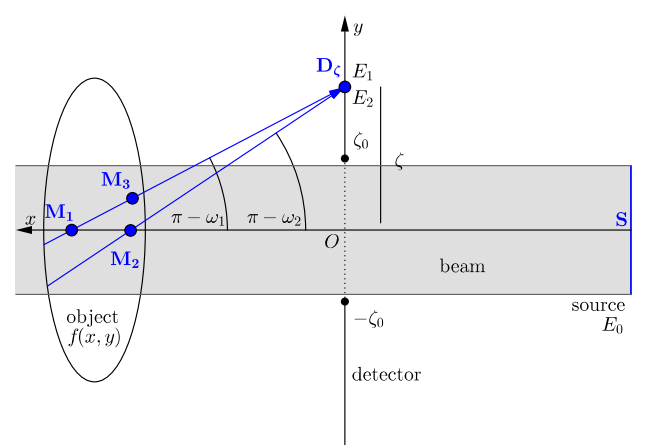

Figure 4. Three positions inside the sample producing scattered radiation captured at a detecting site $\mathbf{D}_{\zeta}$ at different energies $\left\{E_{1}, E_{2}\right\} . \mathbf{M}_{1}$ and $\mathbf{M}_{3}$ having the same scattering angle, generate both energy $E_{1}, \mathbf{M}_{2}$ does the same for energy $E_{2}$.

bution, over all locations where photons are scattered by the same angle and arriving at the same detecting point.

In a forward scattering framework, integration must be performed over a pair of half lines in the shape of a V, with fixed axis direction, these so-called V-line Radon transforms were introduced in (Morvidone et al., 2010) and developed in (Truong and Nguyen, 2011, 2015).

For simplicity, call $\bar{\omega}=\pi-\omega$ the supplementary angle of $\omega$. To cover all projections from the sample to the full detector length, we have to consider $\frac{\pi}{2}<\omega<\frac{3 \pi}{2}$ even if intervals $\left[\frac{\pi}{2}, \pi\right]$ and $\left[\pi, \frac{3 \pi}{2}\right]$ are physically equivalents. Therefore, we will have $-\frac{\pi}{2}<\bar{\omega}<\frac{\pi}{2}$.

The half-space Radon transform, represented by $R^{*}$, is then defined in this paper, for $-\infty<\zeta<\infty$ and $-\frac{\pi}{2}<\bar{\omega}<$ $\frac{\pi}{2}$, as the integral

$$
R^{*} f(\bar{\omega}, \zeta)=\int_{0}^{\infty} \frac{1}{r} f(r \cos \bar{\omega}, \zeta-r \sin \bar{\omega}) \mathrm{d} r
$$

The factor $\frac{1}{r}$ in the last equation comes from the photometric law or the difference in solid angle from the scattering site to the detecting site. $\mathrm{d} r$ is the measure on the half-line. 


\subsubsection{Image formation}

Let $\mathrm{d} I\left(E_{\omega}, \mathbf{D}_{\zeta}\right)$ represent the recorded scattered photon flux time density. It is the number of photons of energy laying in $E_{\omega} \mathrm{d} \omega$ recorded per unit time at $\mathbf{D}_{\zeta}$. It will incorporate the following parameters :

- $I_{0}$ : the incident photon flux density before the scattering event.

- $\sigma^{2 \mathrm{D}}(\omega)$ : the 2D Klein-Nishina differential crosssection (Klein and Nishina, 1928) at an angle $\omega$.

- $f(\mathbf{M})$ : the electron density at $\mathbf{M}$.

- $\mathrm{d} \Omega\left(\mathbf{M}, \mathbf{D}_{\zeta}\right)$ : the solid angle from $\mathbf{M}$ to $\mathbf{D}_{\zeta}$.

- dM: the elementary length around $\mathbf{M}$ over the halfline.

- $\mathrm{d} \omega$ : the elementary variation of $\omega$.

The solid angle $\mathrm{d} \Omega\left(\mathbf{M}, \mathbf{D}_{\zeta}\right)$ can be seen from Figure 3 to be

$$
\mathrm{d} \Omega\left(\mathbf{M}, \mathbf{D}_{\zeta}\right)=2 \arctan \left(\frac{\tau}{2 r} \cos \bar{\omega}\right),
$$

where $\tau$ is the length of the detecting element located at $\mathbf{D}$ and $r$ the Euclidean distance from $\mathbf{M}$ to $\mathbf{D}$.

If $\tau$ is small enough, then $\mathrm{d} \Omega\left(\mathbf{M}, \mathbf{D}_{\zeta}\right)$ can be approximated by $\frac{1}{r} \tau \cos \bar{\omega}$.

The 2D Klein-Nishina differential cross-section is a function of the scattering angle $\omega$ giving the probability of a photon to be scattered by $\omega$ limited to a bi-dimensional scatter plane. It is given by

$$
\sigma^{2 \mathrm{D}}(\omega)=\frac{1}{2} \pi r_{e}^{2}\left(\frac{E_{\omega}}{E_{0}}\right)^{2}\left(\frac{E_{\omega}}{E_{0}}+\frac{E_{0}}{E_{\omega}}-\sin ^{2} \omega\right),
$$

where $r_{e}$ is the classical electron radius.

Consequently, the scattered photon flux density at $\mathbf{D}_{\zeta}$, given a scattering site $\mathbf{M}$, is given by

$$
\mathrm{d} I\left(E_{\omega}, \mathbf{D}_{\zeta} \mid \mathbf{M}\right)=I_{0} \sigma^{2 \mathrm{D}}(\omega) \mathrm{d} \Omega\left(\mathbf{M}, \mathbf{D}_{\zeta}\right) f(\mathbf{M}) \mathrm{d} \omega \mathrm{d} \mathbf{M} .
$$

The recorded scattered flux time density $\mathrm{d} I\left(E_{\omega}, \mathbf{D}_{\zeta}\right)$ recorded at $\mathbf{D}_{\zeta}$ is the integral over all scattering sites laying on the half-line that starts at $\mathbf{D}_{\zeta}$ towards the object with slope $\tan \bar{\omega}$ noted $L_{\omega, \zeta}$. It is hence given by the integral

$$
\mathrm{d} I\left(E_{\omega}, \mathbf{D}_{\zeta}\right)=\int_{\mathbf{M} \in L_{\omega, \zeta}} \mathrm{d} I\left(E_{\omega}, \mathbf{D}_{\zeta} \mid \mathbf{M}\right) .
$$

From the last integral, we can extract the half-space Radon transform, and we are able to express the scattered photon flux time density for photons laying in a recorded energy $E_{\omega} \mathrm{d} \omega$ as

$$
\mathrm{d} I\left(E_{\omega}, \mathbf{D}_{\zeta}\right)=\tau I_{0} \cos \bar{\omega} \sigma^{2 \mathrm{D}}(\omega) R^{*} f(\bar{\omega}, \zeta) \mathrm{d} \omega .
$$

\subsection{Inverse problem : Object Reconstruction}

\subsubsection{Inversion of the HRT}

In order to obtain an inverse formula for (2), we make use of Fourier techniques developed in (Morvidone et al., 2010) related to the V-line Radon transform.

Let $f(x, y)$ be expressed using its $y$-Fourier transform, noted $\hat{f}(x, q)$, as

$$
f(x, y)=\int_{-\infty}^{\infty} \hat{f}(x, q) \mathrm{e}^{2 \pi i y q} \mathrm{~d} q .
$$

Then (2) takes the form

$$
\begin{aligned}
R^{*} f(\bar{\omega}, \zeta)=\int_{0}^{\infty} \frac{1}{r} \int_{-\infty}^{\infty} \hat{f}(r \cos \bar{\omega}, q) \\
\quad \mathrm{e}^{2 \pi i q(\zeta-r \sin \bar{\omega})} \mathrm{d} q \mathrm{~d} r,
\end{aligned}
$$

and applying Fubini's theorem,

$$
\begin{aligned}
R^{*} f(\bar{\omega}, \zeta)=\int_{-\infty}^{\infty} \mathrm{e}^{2 \pi i q \zeta} \int_{0}^{\infty} \frac{1}{r} \hat{f}(r \cos \bar{\omega}, q) \\
\\
\mathrm{e}^{-2 \pi i q r \sin \bar{\omega}} \mathrm{d} r \mathrm{~d} q
\end{aligned}
$$

from where we can extract the $\zeta$-Fourier transform of $R^{*} f(\bar{\omega}, \zeta)$ by writing

$$
\widehat{R^{*} f}(\bar{\omega}, q)=\int_{0}^{\infty} \frac{1}{r} \hat{f}(r \cos \bar{\omega}, q) \mathrm{e}^{-2 \pi i r q \sin \bar{\omega}} \mathrm{d} r .
$$

With a change of variables $x=r \cos \bar{\omega}, t=\tan \bar{\omega}$ and defining the function $g:(t, q) \mapsto \widehat{R^{*} f}(\bar{\omega}, q)$, (11) becomes

$$
g(t, q)=\int_{0}^{\infty} \frac{1}{x} \hat{f}(x, q) \mathrm{e}^{-2 \pi i q t x} \mathrm{~d} x,
$$

which is the Fourier transform of the function $x \mapsto$ $\frac{1}{x} \hat{f}(x, q)$ evaluated at $q t$. One can now apply the inverse formula and the scaling property of Fourier transforms to get $\hat{f}(x, q)$ in the form

$$
\hat{f}(x, q)=|q| x \int_{-\infty}^{\infty} g(t, q) \mathrm{e}^{2 \pi i q t x} \mathrm{~d} t .
$$

Finally, $f(x, y)$ can be reconstructed for all points $(x, y)$ in the object through the $q$-inverse Fourier transform of the last expression.

\subsubsection{Filtered back-projection}

As the classical Radon Transform on straight lines, the filtered back-projection inversion on on a half-space combines the back-projection operation and a filtering operation. It says that the back-projection operator $b_{\omega}(x, y)$ at 
$(x, y)$ for an scattering angle $\omega$ assigns to $(x, y)$ the value of the projection recorded at $\zeta=y+x \tan \bar{\omega}$ where $(x, y)$ was projected, recalling that $\bar{\omega}=\pi-\omega$. That is to say, the back-projection operator is defined by

$$
b_{\omega}(x, y)=R^{*} f(\bar{\omega}, y+x \tan \bar{\omega}) .
$$

A first rough reconstruction of the object can be done by applying the back-projection operator without filtering. The object can then be reconstructed by

$$
f(x, y)=\int_{0}^{\pi / 2} b_{\omega}(x, y) \mathrm{d} \bar{\omega} .
$$

The filtering operation is applied to the $\zeta$-Fourier transform of $R^{*} f(\bar{\omega}, \zeta)$. To obtain the correct filter, we can write the inversion formula of (2) from (8), (13) and the Fubini's theorem as

$$
f(x, y)=x \int_{-\infty}^{\infty} \int_{-\infty}^{\infty}|q| g(t, q) \mathrm{e}^{2 \pi i q(y+x t)} \mathrm{d} q \mathrm{~d} t,
$$

where we identify the inverse Fourier transform of the function $q \mapsto|q| g(t, q)$ evaluated at $y+x t$ by writing

$$
f(x, y)=x \int_{-\infty}^{\infty} \mathscr{F}^{-1}(|q| g)(t, y+x t) \mathrm{d} t,
$$

where $\mathscr{F}^{-1}$ is the inverse Fourier transform.

Finally, in terms of the angle $\bar{\omega}$, last inversion formula reads

$$
f(x, y)=x \int_{-\pi / 2}^{\pi / 2} \mathscr{F}^{-1}\left(|q| \widehat{R^{*} f}\right)(\bar{\omega}, y+x \tan \bar{\omega}) \frac{\mathrm{d} \bar{\omega}}{\cos ^{2} \bar{\omega}} .
$$

The last inverse Fourier transform is called the filtered projection of $R^{*} f$ using the so-called ramp filter $|q|$, it is represented by

$$
\widetilde{R^{*} f}(\bar{\omega}, \zeta)=\mathscr{F}^{-1}\left[|q| \widehat{R^{*} f}\right](\bar{\omega}, \zeta),
$$

and then one is able to write the back-projection operator related to this filtered projection as

$$
\tilde{b}_{\omega}(x, y)=\widetilde{R^{*} f}(\bar{\omega}, y+x \tan \bar{\omega}),
$$

to finally be able to write a filtered back-projection reconstruction of $f(x, y)$ for all $(x, y)$ in the object under a compact form via the integral

$$
f(x, y)=x \int_{-\pi / 2}^{\pi / 2} \tilde{b}_{\omega}(x, y) \frac{\mathrm{d} \bar{\omega}}{\cos ^{2} \bar{\omega}}
$$

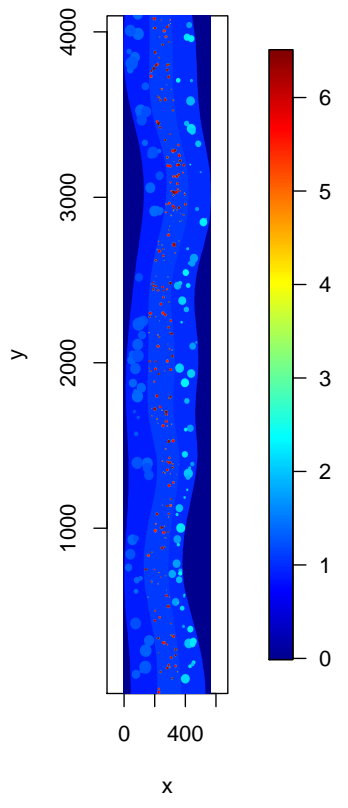

Figure 5. Test sample: A flat stratigraphic phantom.

\section{Simulation of the new CST modality}

A numerical phantom corresponding to a transversal section of a flattened stratigraphic sample is generated and reconstructed in Figs. 5 and 6 respectively via inversion formula (21).

The phantom presents three layers of about $150 \times 4096$ square micrometers with electron densities of $0.9,1.1$ and 1.0 respectively. Grains of random diameter and position were inserted inside the layers, having densities of $1.3 \pm$ $.1,6.0 \pm 0.5$ and $2.0 \pm 0.3$ respectively.

In these first attempts, attenuation and multiple scattering are neglected.

\subsection{Energy resolution of the detector}

Simulation of this new CST modality requires to consider a realistic energy-resolved detector. Let $\Delta E$ be the energy resolution of the detector. Hence, for a fixed detector, we will have a limited number of energy channels recording photons laying on a specific energy range.

Let $C_{i}$ be the energy channel detecting all photons laying on the energy range $\left[E_{i}, E_{i}+\Delta E\right]$. It can be equivalently defined in terms of the scattering angle through (1) for $\omega$ laying in $C_{i}=\left[\omega_{i}, \omega_{i+1}\right]$.

The measured intensity into a given energy channel for a given detecting site is then given by integrating the scattered intensity over that channel

$$
I\left(C_{i}, \mathbf{D}_{\zeta}\right)=\int_{C_{i}} I\left(E, \mathbf{D}_{\zeta}\right) \mathrm{d} E
$$

where $\mathrm{d} I\left(E, \mathbf{D}_{\zeta}\right)$ is given in (7). 
The trapezoidal rule was used to compute numerically last integral with an angular step corresponding to $5 \mathrm{eV}$ by means of (1). Consequently, the same parameters are used for integral (21) with a simple change of variables from $\omega$ to $E$ through (1). Two energy resolutions are considered in simulations, namely $\Delta E \in\{50,100\} \mathrm{eV}$.

\subsection{Spatial discretization}

The unit length considered is $2 \mu \mathrm{m}$. Each pixel in image representations will represent then $2 \times 2 \mu \mathrm{m}^{2}$.

A parallel X-ray beam of $8 \mu \mathrm{m}$ of width crosses a hole in the detector of $12 \mu \mathrm{m}$ of width, hence we have $\zeta_{0}=$ $6 \mu \mathrm{m}$. The medium considered has $512 \times 4096 \mu \mathrm{m}^{2}$ and then we need 512 vertical translations due to the height of the sample to cover the full phantom. The considered detector located at $2 \mu \mathrm{m}$ of the sample over the $O y$-axis has a vertical length of $2048 \mu \mathrm{m}$ including the hole. Thereby, we have 1024-24 detecting sites. (24 sites not considered due to the hole.)

Figure 7 shows the bottom slice of the sample $(8 \mu \mathrm{m}$ of heigh corresponding to the beam width) and its associated image formation through a discretization of (2).

Numerical resolution of integral (2) is performed via the trapezoidal rule with a discretization spatial step $\mathrm{d} r=2$ $\mu \mathrm{m}$.

\subsection{Window functions}

Window functions $\mathrm{w}(q)$ are added to the ramp filter in numerical reconstructions to control high frequencies. Filtered projections related to $R^{*} f$ written in (19) are then rewritten in the form

$$
\widetilde{R^{*} f}(\bar{\omega}, \zeta)=\mathscr{F}^{-1}\left[|q| \mathrm{w}(q) \widehat{R^{*} f}\right](\bar{\omega}, \zeta) .
$$

Cosine related window functions are widely used, they have the form

$$
\mathrm{w}(q)=\cos ^{n}(\pi q) .
$$

Within this family of windows, the case $n=2$ corresponds to the well characterised Hann Window function. During our simulation work we tested various values of $n$ and found out that $n=8$ was the one minimising the mean square error between the object and its reconstruction given the numerical noise produced by the simulation. Still different values of $n$ gives satisfactory reconstructions as well. Horizontal artefacts in reconstructions are due to vertical translations of the object. Grains inside the matrix layers are reconstructed at the correct depth, size and relative densities, without the need of a relative rotation between phantom and detector.

\section{Conclusions and perspectives}

A new X-ray imaging modality based on Compton scattering is proposed, the particular aim is a 3D reconstruction of flat objects without relaying on a relative rotation between the studied sample and the imaging setup. Modelling of both image formation by means of the half-space
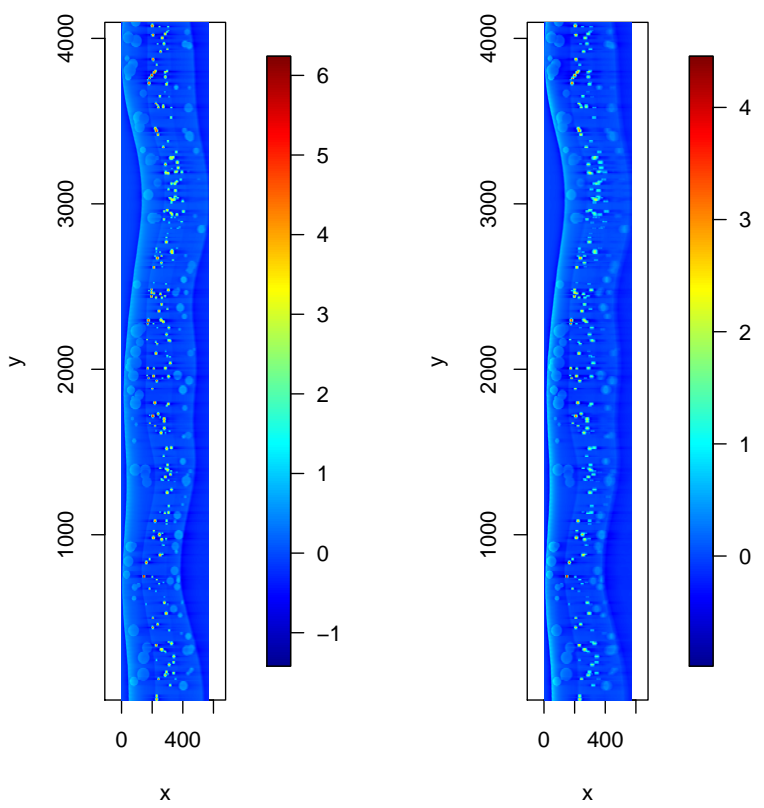

(a) Reconstruction with $\Delta E=50$ (b) Reconstruction with $\Delta E=$ $\mathrm{eV}$. $100 \mathrm{eV}$.

Figure 6. Numerical results of reconstructions from backscattered data.

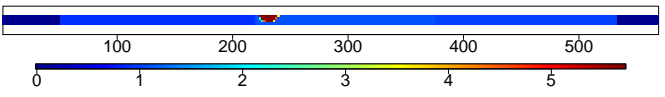

(a) Bottom $8 \mu \mathrm{m}$ of the phantom.

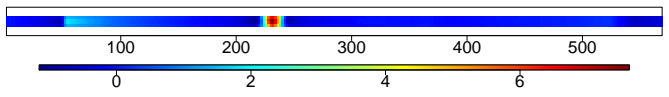

(b) Reconstruction of Figure 7a with $\Delta E=50$.

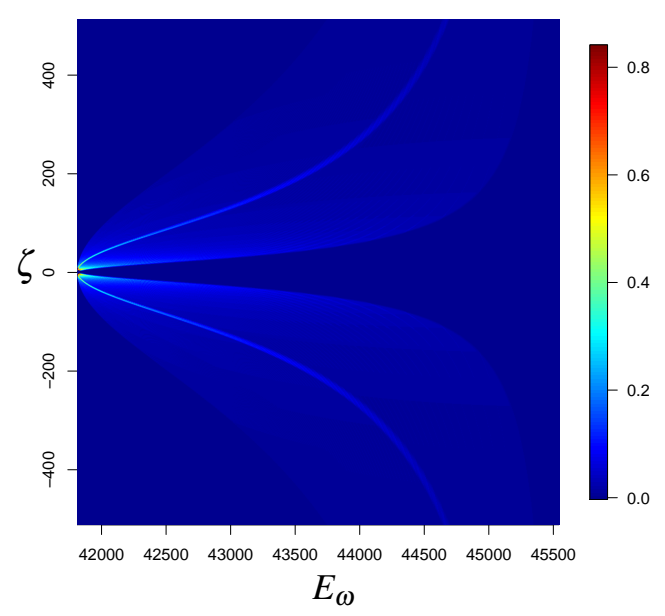

(c) HRT of object shown in Figure 7a. We can recognise the effect of the middle red grain at the two highlighted curves.

Figure 7. Image formation process through this new modality. 
Radon transform and object reconstruction by a filtered back-projection inversion are presented, considering realistic energy resolved detectors, and supported with numerical simulations proving feasibility with experimental data. Simulations of the three-dimensional setup, regarding a conical Radon transform, are in progress for both forward and backward scattering. Yet the present results are already very encouraging considering the problem of non-destructive and non-invasive 3D imaging of samples supported by a deep or dense material.

\section{Acknowledgment}

The authors would like to thank Pierre Gueriau and Mathieu Thoury for their support, discussions and help to acquire images of heritage objects. Annelies van Loon for providing the image of the Rembrandt painting, and the field workers who collected the fossil actinopterygian.

Patricio Guerrero would also like to thank the Fondation des Sciences du Patrimoine for providing a $\mathrm{PhD}$ grant to support his research work.

\section{References}

J. Cebeiro, M. Morvidone, and M. K. Nguyen. Back-projection inversion of a conical Radon transform. Inverse Problems in Sciences and Engineering, 24(2):328-352, April 2015.

P. Gueriau, C. Mocuta, D. B. Dutheil, S. X. Cohen, D. Thiaudière, S. Charbonnier, G. Clémént, and L. Bertrand. Trace elemental imaging of rare earth elements discriminates tissues at microscale in flat fossils. Plos One, 9(1):e86946, 2014.

O. Klein and Y. Nishina. The scattering of light by free electrons according to dirac's new relativistic dynamics. Nature, 122 (3072):398-399, 1928.

M. Morvidone, M. K. Nguyen, T. T. Truong, and H. Zaidi. On the V-line radon transform and its imaging applications. International Journal of Biomedical Imaging, 2010:11, 2010.

M. K. Nguyen, T. T. Truong, and P. Grangeat. Radon transforms on a class of cones with fixed axis direction. Journal of Physics A: Mathematical and General, 38(37):8003, 2005.

S. J. Norton. Compton scattering tomography. Journal of Applied Physics, 76(4):2007-2015, 1994.

J. Radon. Berichte über die verhandlungen der königlich sächsischen gesellschaft der wissenschaften zu leipzig. Mathem. Phys. Klasse, 69:262-266, 1917.

T. T. Truong and M. K. Nguyen. On new V-line radon transforms in $\mathbb{R}^{2}$ and their inversion. Journal of Physics A: Mathematical and Theoretical, 44(7):075206, 2011.

T. T. Truong and M. K. Nguyen. New properties of the Vline radon transform and their imaging applications. Journal of Physics A: Mathematical and Theoretical, 48(40):405204, 2015. 